University of Wollongong

Research Online

Faculty of Engineering and Information

Faculty of Engineering and Information

Sciences - Papers: Part B

Sciences

2018

Pairing-dependent superconductivity gap and nonholonomic Andreev reflection in Weyl semimetal/Weyl superconductor heterojunctions

Jun Fang

Peking University

Wenye Duan

Peking University, wduan@uow.edu.au

Junfeng Liu

South University of Science and technology of China

C Zhang

University of Wollongong, czhang@uow.edu.au

Zhongshui Ma

Peking University, zma@uow.edu.au

Follow this and additional works at: https://ro.uow.edu.au/eispapers1

Part of the Engineering Commons, and the Science and Technology Studies Commons

Research Online is the open access institutional repository for the University of Wollongong. For further information contact the UOW Library: research-pubs@uow.edu.au 


\title{
Pairing-dependent superconductivity gap and nonholonomic Andreev reflection in Weyl semimetal/Weyl superconductor heterojunctions
}

\author{
Abstract \\ We study superconductivity states mediated by the BCS and Fulde-Ferrell-Larkin-Ovchinnikov (FFLO) \\ pairings in superconducting Weyl semimetals. It is found that a mixture of BCS and FFLO pairings results \\ in a distinctive double-gap structure for superconducting states. With a heterojunction of a Weyl \\ semimetal and a superconducting Weyl semimetal, we demonstrate the nonholonomic Andreev reflection \\ and show that the intra- and internode Andreev reflections increase at the edges of the effective gap. The \\ influence of interface potentials on the Andreev reflections is investigated. The conductance spectra \\ arising from the mixed superconducting pairings is also analyzed. \\ Disciplines \\ Engineering | Science and Technology Studies

\section{Publication Details} \\ Fang, J., Duan, W., Liu, J., Zhang, C. \& Ma, Z. (2018). Pairing-dependent superconductivity gap and \\ nonholonomic Andreev reflection in Weyl semimetal/Weyl superconductor heterojunctions. Physical \\ Review B: Covering condensed matter and materials physics, 97 (16), 165301-1-165301-9.
}




\title{
Pairing-dependent superconductivity gap and nonholonomic Andreev reflection in Weyl semimetal/Weyl superconductor heterojunctions
}

\author{
Jun Fang, ${ }^{1}$ Wenye Duan, ${ }^{1}$ Junfeng Liu, ${ }^{2}$ Chao Zhang, ${ }^{3}$ and Zhongshui Ma ${ }^{1,4}$ \\ ${ }^{1}$ School of Physics, Peking University, Beijing 100871, China \\ ${ }^{2}$ Department of Physics, South University of Science and Technology of China, Shenzhen 518055, China \\ ${ }^{3}$ School of Physics, University of Wollongong, New South Wales 2522, Australia \\ ${ }^{4}$ Collaborative Innovation Center of Quantum Matter, Beijing 100871, China
}

(Received 16 March 2017; published 4 April 2018)

\begin{abstract}
We study superconductivity states mediated by the BCS and Fulde-Ferrell-Larkin-Ovchinnikov (FFLO) pairings in superconducting Weyl semimetals. It is found that a mixture of BCS and FFLO pairings results in a distinctive double-gap structure for superconducting states. With a heterojunction of a Weyl semimetal and a superconducting Weyl semimetal, we demonstrate the nonholonomic Andreev reflection and show that the intraand internode Andreev reflections increase at the edges of the effective gap. The influence of interface potentials on the Andreev reflections is investigated. The conductance spectra arising from the mixed superconducting pairings is also analyzed.
\end{abstract}

DOI: 10.1103/PhysRevB.97.165301

\section{INTRODUCTION}

The superconductivity in Weyl semimetals (WSMs) was investigated recently [1-3] in order to understand the interplay between superconductivity and nontrivial topology in electronic structures. The first-principles calculation predicted [4] that the $\mathrm{WoTe}_{2}$ material hosts the WSM state with four pairs of Weyl nodes in the bulk band structure. Later, experiments [5] revealed the unconventional superconductivity in the $\mathrm{WoTe}_{2}$ material. Experiment showed that $\mathrm{UPt}_{3}$ exhibits the superconducting phase with the nodal line [6]. The superconducting transition temperature is about $0.5 \mathrm{~K}$. However, a recent study showed that it is a superconducting Weyl semimetal with nodal points [7]. The superconductivity in doped WSMs [1-3,8-13] has become a topic of interest in unconventional superconductivity. Theoretically, for the lightly doped Weyl semimetal, the Fermi energy is sufficiently close to the Weyl nodes such that the Fermi surface consists of an even number of disconnected Fermi sheets around the Weyl nodes. The linear dispersion in the vicinity of these nodes gives rise to low-energy chiral symmetry. In this situation, those topologically nontrivial properties in undoped Weyl semimetals are mostly retained. Unconventional superconductivity has been predicted to occur in doped WSM with inversion symmetry and a topologically nontrivial Fermi surface. Two competing pairing mechanisms are possible for realizing the superconductivity in doped WSMs [1,8], i.e., the internode BCS pairing mechanism [14] and the intranode Fulde-Ferrell-Larkin-Ovchinnikov (FFLO) pairing mechanism $[15,16]$. The BCS pairing occurs between fermions with opposite momenta and forms a zero-momentum Cooper pair. However, the FFLO pairing occurs between fermions with finite total momenta, so that the superconducting order parameter is spatially nonuniform. The BCS paired states are topologically nontrivial with gapless nodes in the energy dispersion, and the FFLO paired state is topologically trivial with a full nodeless gap $[1,3,8,9]$.
Although many works have been devoted to investigating which kind of pairing state is preferred in doped WSM, it has remained unclear which pairing mechanism is favored in a superconducting Weyl semimetal (SWSM). Based on the assumption of only one kind of pairing, the preferred pairing state is identified by comparing the ground-state energy in the BCS pairing with that in the FFLO pairing. References $[1,3]$ argued that the FFLO state has a lower energy. Later, by using the standard mean-field theory [8] and the Bogoliubov-de Gennes (BdG) equations [9], it was demonstrated that the energy of the BCS paired state is lower than that of the FFLO state. These works have focused mainly on the preferred pairing state, either FFLO or BCS, in a SWSM. However, the possibility of a mixture of FFLO and BCS pairings cannot be ignored. It would display competition between topologically nontrivial and topologically trivial superconducting states in a SWSM. Several theoretical works in the literature discuss the transition between two distinct paired states [17]. A mixture of BCS and FFLO pairings may naturally occur in the transition regime from the BCS to the FFLO and vice versa. For a BCS-FFLO transition, it was also pointed out [8] that the FFLO paired state could emerge in a SWSM with an original BCS paired state when the Weyl nodes are shifted to different energies. As long as this energy difference is comparable to the BCS pairing potential, the BCS state would be suppressed, so that the FFLO paired state is preferred. It is desirable to investigate the role of two distinct superconducting correlations in Weyl materials. Because the two kinds of low-energy excitations can be expressed as a superposition of states in two Weyl nodes, the interaction potential provides the possible mixing of two superconducting order parameters which is traceable to two kinds of pairings. When the BCS and the FFLO coexist, it is expected that by varying the relative weighting of the BCS and the FFLO superconducting order parameters, various superconducting states between the BCS and FFLO states can be engineered. In the present work, instead of investigating 
the conversion between the two kinds of superconducting states, we study the transport properties with a mixture of BCS and FFLO pairings. The effective gap function with a mixture of FFLO and BCS pair potentials is calculated phenomenologically.

Hybrid structures of semimetals and superconductors are a suitable platform for studying possible superconducting states. Previous studies on the $s-, p$-, and $d$-wave superconductors have shown that Andreev reflection (AR) [18] in hybrid structures of metals (or semiconductors) and superconductors can reveal information about the pairing symmetry in the system [19]. Therefore, AR can be used to determine the pairing scheme for a given doped SWSM. The signature of the Larkin-Ovchinnikov state in the conductance has been studied in a normal-metal/superconductor junction [20]. For a pure FFLO pairing and a pure BCS pairing, ARs were studied in a WSM/SWSM hybrid structure [21] and in a WSM/normalsuperconductor heterojunction [22]. The measurement of the Josephson effect has been considered a way to detect the existence of unconventional superconducting states [23,24]. Recently, Kim et al. [25] proposed a four-terminal transport Josephson junction of a Weyl semimetal to probe FFLO and nodal BCS states in the superconducting phase of the inversionsymmetric doped WSM. The condition of switching chirality was studied for AR in a magnetic Weyl semimetal [26].

In this paper we investigate the transport properties mediated by the BCS and FFLO pairings in the WSM/SWSM hybrid structures. We construct a phenomenological model of effective pairing potential which includes a mixture of the BCS and FFLO pairings. It is shown that two gaps $\Delta_{\text {eff }}^{( \pm)}=\left|\Delta_{F} \pm \widetilde{\Delta}_{B}\right|$ exist, where $\Delta_{F}$ and $\widetilde{\Delta}_{B}$ are the modified FFLO and BCS pairing order parameters. The existence of two gaps can result in different tunneling characteristics in a WSM/SWSM hybrid with Andreev reflection. To reveal the dependence of superconducting states on the effective pairing potentials, we investigate AR in a doped WSM/SWSM hybrid structure. For incident energies below $\Delta_{\text {eff }}^{(-)}$, both the gaps take effect. However, only $\Delta_{\text {eff }}^{(+)}$takes effect if $\Delta_{\text {eff }}^{(-)}<$ $E<\Delta_{\text {eff }}^{(+)}$; only half of the incident electrons are paired and undergo AR. We refer to such an AR as the nonholonomic AR. The reflection is strongly dependent on the interface potentials. For imperfect interfaces, two kinds of interface potentials are considered in our calculations: the intranode scattering interface potential (intra-SIP) for scattering within the same Weyl node and the internode scattering interface potential (inter-SIP) for scattering between different Weyl nodes. These interface potentials can affect the intranode and internode component of ARs significantly. It is found that the conductance exhibits an oscillatory behavior for the intra-SIP, while it decays exponentially for the inter-SIP.

This paper is organized as follows: In Sec. II, we solve the Bogoliubov-de Gennes (BdG) equations for a doped WSM/SWSM hybrid structure. The general solutions for the coexistence of two superconducting pairings are obtained. In Sec. III, we calculate the transport coefficients with a perfect interface. ARs with a mixture of both BCS and FFLO pairings are discussed. In Sec. IV the boundary conditions for the intra-SIP and the inter-SIP at the interface of a WSM/SWSM heterojunction are introduced. The effects of interface potentials on the transport coefficients are discussed. The intranode and internode components of ARs with energy in the superconducting gap are analyzed. The incident-angledependent conductance and the total conductance are calculated in Sec. V. Section VI summarizes the results.

\section{THE EFFECTIVE SUPERCONDUCTING GAP IN A SWSM WITH A MIXTURE OF FFLO AND BCS PAIRINGS}

We consider a WSM/SWSM heterojunction with its interface at $z=0$. For simplicity, we consider only a pair of Weyl nodes localized at $\mathbf{K}_{ \pm}=(0,0, \pm b)$ in the momentum space. The BdG Hamiltonian can be written in the form

$$
\mathcal{H}_{B d G}=\left(\begin{array}{cc}
H^{(e)}-\mu \tau_{0} & \Theta(z) \Delta \\
\Theta(z) \Delta^{*} & \mu \tau_{0}-H^{(h)}
\end{array}\right)+\delta(z) V
$$

on the basis of the Nambu spinor $\Psi=$ $\left(\psi_{+, \uparrow} \psi_{+, \downarrow} \psi_{-, \uparrow} \psi_{-, \downarrow} \psi_{+, \downarrow}^{\dagger}-\psi_{+, \uparrow}^{\dagger} \psi_{-, \downarrow}^{\dagger}-\psi_{-, \uparrow}^{\dagger}\right)^{T}$, where the plus and minus signs stand for the chirality of Weyl nodes, the up and down arrows stand for the spins, $\Theta(z)$ is a step function $[\Theta(z)=1$ for $z>0$ and 0 for $z<0], \mu$ is the chemical potential, $\Delta$ is the superconducting order parameter in terms of the FFLO and BCS order parameters, $\Delta_{F}(\mathbf{x})=\Delta_{F} e^{ \pm i 2 b z / \hbar}$ and $\Delta_{B}(\mathbf{x})=\Delta_{B}$,

$$
\Delta=\Delta_{F}\left[\tau_{0} \cos \left(\frac{2 b z}{\hbar}\right)+\tau_{x} \frac{\Delta_{B}}{\Delta_{F}}+i \tau_{z} \sin \left(\frac{2 b z}{\hbar}\right)\right] \sigma_{0},
$$

and $H^{(e)}$ and $H^{(h)}$ are the Hamiltonians for electronlike and holelike carriers,

$$
H^{(e)}(\mathbf{p}, b)=v_{F}\left[\tau_{0}\left(\sigma_{x} p_{x}+\sigma_{y} p_{y}-\sigma_{z} b\right)+\tau_{z} \sigma_{z} p_{z}\right]
$$

and

$$
H^{(h)}(\mathbf{p}, b)=T H^{(e)}(\mathbf{p}, b) T^{-1}=H^{(e)}(\mathbf{p},-b),
$$

respectively, with a Fermi velocity $v_{F} . T$ is a time-reversal operator, and $T=i \tau_{0} \sigma_{y} \mathcal{K}$, with $\mathcal{K}$ being complex conjugation. In these expressions, $\sigma_{x}, \sigma_{y}$, and $\sigma_{z}$ are the Pauli matrices describing the spins; $\tau_{x}, \tau_{y}$, and $\tau_{z}$ are the Pauli matrices describing the chirality in the space spanned by two Weyl nodes; and $\sigma_{0}$ and $\tau_{0}$ are $2 \times 2$ identity matrices. In addition, we introduce an interface potential $V$ in Eq. (1) to describe the proximity effect at the interface. Our model conserves the inversion symmetry $\Lambda \mathcal{H}_{B d G}(\mathbf{p}, \mathbf{r}) \Lambda^{-1}=$ $\mathcal{H}_{B d G}(-\mathbf{p},-\mathbf{r})$, where $\Lambda=I_{2 \times 2} \otimes P$, with $P=\tau_{x} \sigma_{z}$, where $I_{2 \times 2}$ is a $2 \times 2$ identity matrix for the electron and hole. The time reversal is manifested in terms of the Hamiltonian as $[27,28] T H_{I}^{(e)}\left(\mathbf{p}^{*}, b\right) T^{-1}=H_{I}^{(e)}(-\mathbf{p},-b)$, where $\mathbf{p}$ is an operator, while a complex conjugate of the Hamiltonian is embodied in the operator $T$. The change $b \rightarrow-b$ implies that the time-reversal symmetry is not preserved. For the BdG Hamiltonian we found $\mathcal{T} \mathcal{H}_{B d G}\left(\mathbf{p}^{*}, b\right) \mathcal{T}^{-1}=\mathcal{H}_{B d G}(-\mathbf{p},-b)$ $\left[\mathcal{T} \mathcal{H}_{B d G}(\mathbf{p}, b) \mathcal{T}^{-1}=\mathcal{H}_{B d G}(\mathbf{p},-b)\right]$. Our Hamiltonian is invariant under charge-conjugation (or particle-hole) symmetry with $\mathcal{C H}_{B d G}\left(\mathbf{p}^{*}, b\right) \mathcal{C}^{-1}=-\mathcal{H}_{B d G}(-\mathbf{p}, b)\left[\left\{\mathcal{C}, \mathcal{H}_{B d G}(\mathbf{p}, b)\right\}=\right.$ $0,\{\cdots\}$ is an anticommuting relation], where $\mathcal{C}=\lambda_{y} \tau_{0} \sigma_{y} K$ is the charge-conjugation (particle-hole) operator and $\lambda_{y}$ is a Pauli matrix for the electron-hole degree of freedom. The chiral symmetry is represented by the operator $[27,28] \mathcal{S}=\mathcal{T C}$, a combination of particle-hole and time-reversal symmetries. It is found that $\mathcal{S} \mathcal{H}_{B d G}(\mathbf{p}, b) \mathcal{S}^{-1}=-\mathcal{H}_{B d G}(\mathbf{p},-b)$. The change 
in Weyl nodes under $\mathcal{S}$ reflects the fact that the Weyl superconductor has chiral symmetry with a pair nodes of opposite chirality.

The Hamiltonian can be transformed into another form under the unitary transformation $\mathcal{U}=I \otimes \Sigma, \mathcal{U H}_{B d G} \mathcal{U}^{-1}=$ $\mathcal{H}_{B d G}^{\prime}$, where $\Sigma=(1 / 2)\left[\tau_{0}\left(\sigma_{0}-i \sigma_{z}\right)+\tau_{z}\left(\sigma_{0}+i \sigma_{z}\right)\right]$, with $\Sigma \Sigma^{\dagger}=\tau_{0} \sigma_{0} . \mathcal{H}_{B d G}^{\prime}$ has the same form as $\mathcal{H}_{B d G}$ in Eq. (1), but $H^{\prime(e)}(\mathbf{p}, b)=v_{F}\left[\tau_{z}\left(\sigma_{x} p_{x}+\sigma_{y} p_{y}+\sigma_{z} p\right)_{z}-\tau_{0} b\right]$, and $H^{\prime(h)}(\mathbf{p}, b)=T H^{(e)}(\mathbf{p}, b) T^{-1}=H^{\prime(e)}(\mathbf{p},-b)$, as given in Ref. [28]. Taking the BCS pairing as an example, the superconducting order parameter becomes $\Delta^{\prime}=\Delta_{B} \sigma_{z} \tau_{y}$, which is consistent with that in Ref. [29]. Because the Hamiltonians $\mathcal{H}_{B d G}$ and $\mathcal{H}_{B d G}^{\prime}$ are connected by a unitary operator $\mathcal{U}$, they depend only on the general properties of the WSM and SWSM under consideration along with the symmetries of the unitary operation. The inversion symmetry is imposed by $\Lambda^{\prime} \mathcal{H}_{B d G}^{\prime}(\mathbf{p}, \mathbf{r}) \Lambda^{\prime-1}=\mathcal{H}_{B d G}^{\prime}(-\mathbf{p},-\mathbf{r})$, where $\Lambda^{\prime}=$ $\mathcal{U} \Lambda \mathcal{U}^{-1}=I_{2 \times 2} \otimes P^{\prime}$, with $P^{\prime}=\tau_{y} \sigma_{0}$. It is interesting to note that $\mathcal{U} \mathcal{T H}_{B d G} \mathcal{T}^{-1} \mathcal{U}^{-1}=\mathcal{T} \mathcal{U} \mathcal{H}_{B d G} \mathcal{U}^{-1} \mathcal{T}^{-1}=\mathcal{T} \mathcal{H}_{B d G}^{\prime} \mathcal{T}^{-1}$ due to the time-reversal operator $\mathcal{T}$ commuting with $\mathcal{U}, \mathcal{U} \mathcal{T}=\mathcal{T U}$. Therefore, the time-reversal characteristics of the system remain unchanged. In addition, the time reversal can also be represented as the switch from $\mathbf{p}$ to $-\mathbf{p}$ together with the exchange of the two nodes. The time-reversal operator is expressed in the form $\tau_{x} T$ up to a unitary rotation $\tau_{x}: H^{\prime(e)}(-\mathbf{p},-b)=$ $\tau_{x} T \mathcal{H}^{\prime(e)}(\mathbf{p}, b) T^{-1} \tau_{x}=T \tau_{x} \Sigma H^{(e)} \Sigma^{-1} \tau_{x} T^{-1}, \quad$ so that $\left(I_{2 \times 2} \otimes \tau_{x}\right) \mathcal{T} \mathcal{H}_{B d G}^{\prime}(\mathbf{p}, b) \mathcal{T}^{-1}\left(I_{2 \times 2} \otimes \tau_{x}\right)=\mathcal{H}_{B d G}^{\prime}(-\mathbf{p},-b)$.

Under this unitary rotation the charge-conjugation symmetry is represented as $\mathcal{C}^{\prime} H_{B d G}^{\prime} \mathcal{C}^{\prime-1}=\mathcal{C} U^{\prime} H_{B d G} U^{\prime-1} \mathcal{C}^{-1}$, where $\mathcal{C}^{\prime}=\lambda_{y} \tau_{x} \sigma_{y} K$ and $U^{\prime}=\left(I_{2 \times 2} \otimes \tau_{x}\right) U$. The chiral symmetry becomes $\mathcal{S}^{\prime} \mathcal{H}_{B d G}(\mathbf{p}, b) \mathcal{S}^{\prime-1}=-\mathcal{H}_{B d G}(-\mathbf{p},-b)$ along with the switch from $\mathbf{p}$ to $-\mathbf{p}$.

We now solve the BdG equation in the WSM and the SWSM separately. In the region of the WSM $(z<0), \Delta=0$, the eigenvalues of Hamiltonian (1) are found as

$$
E_{\eta, \gamma, \kappa}^{W}=\eta\left(\kappa v_{F} p_{\gamma}-\mu\right)
$$

where $\kappa= \pm$ label the conduction and valance bands, $\eta= \pm$ indicate the electron and hole, and $p_{\gamma}=\sqrt{p_{\|}^{2}+p_{z, \gamma}^{2}}$, with subscripts $\gamma= \pm$ for the two Weyl points located at $\pm \mathbf{K}, p_{\|}=$ $\sqrt{p_{x}^{2}+p_{y}^{2}}$, and $p_{z, \gamma}=p_{z}-\gamma b$.

In the region of the SWSM $(z>0)$ the Bogoliubov Hamiltonian $\mathcal{H}^{B d G} \Psi=E \Psi$ can be diagonalized in the representation of the phase-severed quasiparticle wave function

$$
\begin{aligned}
\Psi= & \left(u_{+, \uparrow} e^{b z / \hbar} u_{+, \downarrow} e^{b z / \hbar} v_{-, \uparrow} e^{-b z / \hbar} v_{-, \downarrow} e^{-b z / \hbar} u_{+, \downarrow}^{*} e^{-b z / \hbar}\right. \\
& \left.-u_{+, \uparrow}^{*} e^{-b z / \hbar} v_{-, \downarrow}^{*} e^{b z / \hbar}-v_{-, \uparrow}^{*} e^{b z / \hbar}\right)^{T}
\end{aligned}
$$

The quasiparticle energy is found as

$$
\begin{aligned}
& E_{\eta, \gamma, \kappa}^{S(\beta)} \\
& =\kappa \eta \sqrt{\left(\sqrt{v_{F}^{2} p_{\gamma}^{2}+\Delta_{F}^{2}-\Delta_{\mathrm{eff}}^{(\beta) 2}}-\kappa \sqrt{\Delta_{B}^{2}+\mu^{2}}\right)^{2}+\Delta_{\mathrm{eff}}^{(\beta) 2}},
\end{aligned}
$$

where

$$
\Delta_{\mathrm{eff}}^{(\beta)}=\left|\Delta_{B} \sqrt{\frac{v_{F}^{2} p_{\|}^{2}}{\Delta_{B}^{2}+\mu^{2}}}+\beta \Delta_{F} \frac{\mu}{\sqrt{\Delta_{B}^{2}+\mu^{2}}}\right| .
$$

$\Delta_{\text {eff }}^{(\beta)}$ is a function of $\Delta_{F}, \Delta_{B}$, and $p_{\|}$. This expression shows that the co-occurrence of the BCS and FFLO pairings induces one more degree of freedom, $\beta= \pm$. Accordingly, the spectra $E_{\eta, \gamma, \kappa}^{S(\beta)}$ exhibit the structure with an effective gap $\Delta_{\text {eff }}^{(\beta)}$. The term with $\beta$ is traceable to the broken translational invariance for the FFLO pairing potential. The $\beta$ term plays a significant role in tuning the effective pairing between the BCS and FFLO pairings for electrons from Weyl nodes $K_{ \pm}$.

In the case that only the FFLO pairing exists, $\Delta_{\text {eff }}^{(\beta)}$ reduces to $\Delta_{F} . E_{\eta, \gamma, \kappa}^{S, F}$ becomes $\kappa \eta \sqrt{\left(\mu-\kappa v_{F} p_{\gamma}\right)^{2}+\Delta_{F}^{2}}$. This energy spectrum is fully gapped with a width of $2 \Delta_{F}$. However, for the case of pure BCS pairing, the energy spectra in Eq. (7) reduce to

$$
E_{\eta, \gamma, \kappa}^{S, B}=\kappa \eta \sqrt{\widetilde{\Delta}_{B}^{2}+\left(\sqrt{\mu^{2}+\Delta_{B}^{2}-\widetilde{\Delta}_{B}^{2}}-\kappa v_{F} p_{\gamma}\right)^{2}},
$$

where $\tilde{\Delta}_{B}=\Delta_{B} \sin \theta$ and $\theta=\arccos \left(p_{z, \gamma} / p_{\gamma}\right) . \theta$ is half of the angle between the spin orientations of two electrons in a Cooper pair. It is shown that $E_{\eta, \gamma, \kappa}^{S, B}$ has a $\theta$-dependent gap $2 \widetilde{\Delta}_{B} \cdot \theta=0$ and $\pi$ correspond to the momentum along the positive $z$ direction and the negative $z$ direction, respectively. The spins for the electrons in these two orientations at nodes $\mathbf{K}_{+}$and $\mathbf{K}_{-}$are parallel. Consequently, there is no BCS paired, so that $\widetilde{\Delta}_{B}=0$ at $\theta=0$ and $\pi$. The appearance of bulk gapless states indicates its topological nontriviality for the SWSM with the BCS pairing. These two zero-gap nodes in each of the Weyl nodes reflect that four new nodal points, located at the points $\mathbf{Q}_{ \pm}^{\mathbf{K}_{\gamma}}=\left(0,0, \gamma b \pm \sqrt{\mu^{2}+\Delta_{B}^{2}} / v_{F}\right)$, are induced by a BCS pairing [9]. We define $\tilde{\mu}=\sqrt{\mu^{2}+\Delta_{B}^{2} \cos ^{2} \theta}$ as an effective chemical potential. Because $\widetilde{\mu}$ depends on the spin orientations in pairing, we found that the spin orientations in the BCS pairing not only affect the gap in the energy spectrum but also create a shift in the chemical potential.

Because $\mu \gg \Delta_{B / F}$ and $v_{F} p_{\gamma} \sim \mu$ around Fermi energy, $E_{\eta, \gamma, \kappa}^{S(\beta)}$ can be approximately written as $E_{\eta, \gamma, \kappa}^{S(\beta)}=$ $\kappa \eta \sqrt{\left(v_{F} p_{\gamma}-\kappa \mu\right)^{2}+\Delta_{\text {eff }}^{(\beta) 2}}$. The effective gap function is written as $\Delta_{\text {eff }}^{(\beta)} \simeq\left|\widetilde{\Delta}_{B}+\beta \Delta_{F}\right|$. Two gaps exist for a mixture of BCS and FFLO pairings. Such properties of the excitation spectrum differ qualitatively from those obtained with a pure BCS pairing as well as a pure FFLO pairing superconducting state. We show the spectra in Fig. 1(a), in which the curved cerise and ultramarine surfaces are, respectively, for $\beta=+$ and $\beta=-$. The gaps $\Delta_{\text {eff }}^{(+)}$and $\Delta_{\text {eff }}^{(-)}$are exhibited. Different from $\Delta_{\text {eff }}^{(+)}, \Delta_{\text {eff }}^{(-)}$can vanish when $\Delta_{F}=\Delta_{B} \sin \theta$. For the purpose of illuminating the structure of the effective gap for a mixture of both BCS and FFLO pairings, we plot $\Delta_{\text {eff }}^{(+)} \simeq\left|\widetilde{\Delta}_{B}+\Delta_{F}\right|$ and $\Delta_{\text {eff }}^{(-)} \simeq\left|\widetilde{\Delta}_{B}-\Delta_{F}\right|$ as functions of $\Delta_{F}$ and $\theta \in[0, \pi]$ in Figs. 1(b) and 1(c), respectively, where the relation $\Delta_{B}+\Delta_{F}=1.5 \Delta_{0}$ is assumed. A nodal ring appears in $\Delta_{\text {eff }}^{(-)}$and takes shape of $\Delta_{F}=1.5 \Delta_{0} \sin \theta /(1+\sin \theta)$. The cross-sectional views of $\Delta_{\text {eff }}^{(+)}$and $\Delta_{\text {eff }}^{(-)}$at $\Delta_{0}$ are shown in 

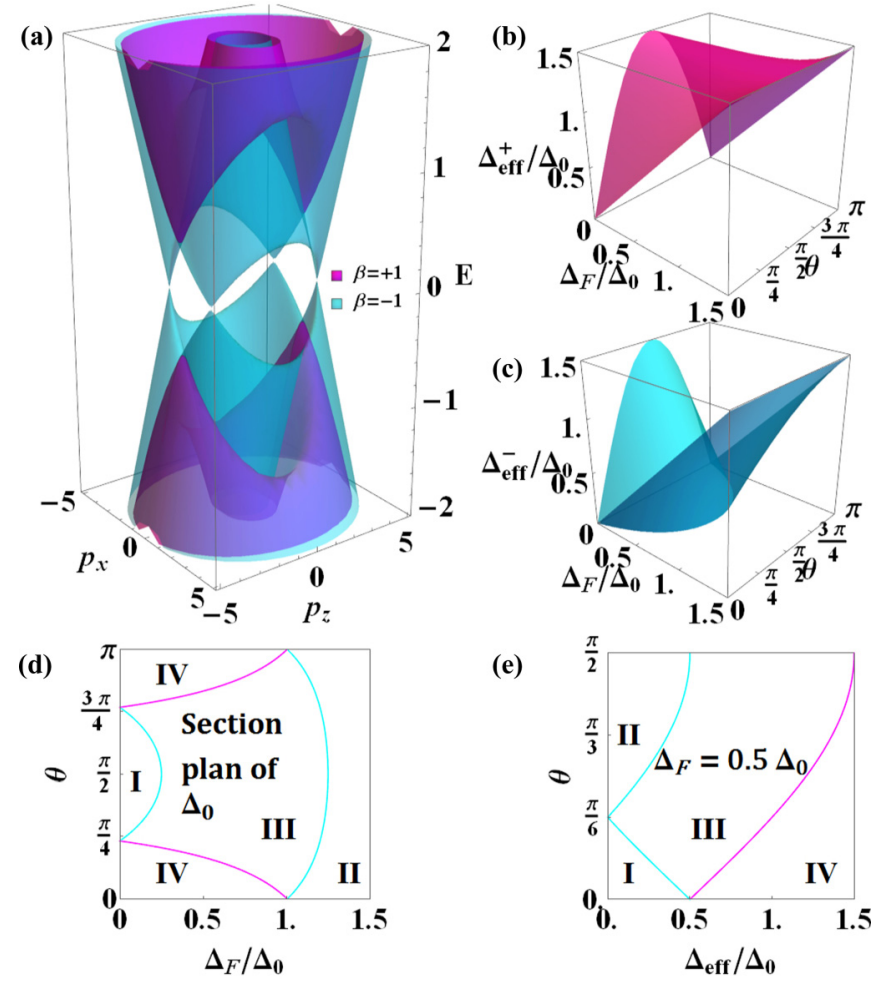

FIG. 1. (a) The spectra of a SWSM with a mixture of BCS and FFLO pairings, in which the curved cerise and ultramarine surfaces are, respectively, for $\beta=+1$ and $\beta=-1$. (b) and (c) The general view of the effective superconducting gaps $\Delta_{\text {eff }}^{(+)}$and $\Delta_{\text {eff }}^{(-)}$, where $\Delta_{F}+\Delta_{B}=1.5 \Delta_{0}$ is used in the calculation. (d) The cross-sectional views of $\Delta_{\text {eff }}^{( \pm)}$at the value $\Delta_{0}$. (e) Effective gap vs $\theta$ at $\Delta_{F}=0.5 \Delta_{0}$.

Fig. 1(d). The lines of demarcation in Fig. 1(d) are given by the cerise curve, $(1.5 \sin \theta-1) \Delta_{0}+\Delta_{F}(1-\sin \theta)=0$, and the ultramarine curves, $(1.5 \sin \theta-1) \Delta_{0}-(1+\sin \theta) \Delta_{F}=$ 0 and $(1.5 \sin \theta+1) \Delta_{0}-(1+\sin \theta) \Delta_{F}=0$. The effective gap has four regions defined by $\theta$ and the relative partition between $\Delta_{F}$ and $\widetilde{\Delta}_{B}$. In region $\mathrm{I}, \widetilde{\Delta}_{B}>\Delta_{F}$, which is segregated from region III by $\widetilde{\Delta}_{B}-\Delta_{F}$. In region II, $\Delta_{F}>\widetilde{\Delta}_{B}$, which is segregated from region III by $\Delta_{F}-\widetilde{\Delta}_{B}$. Region IV is a normal WSM which is segregated from region III by $\widetilde{\Delta}_{B}+\Delta_{F}$. $\widetilde{\Delta}_{B}$ and $\Delta_{F}$ are well matched in region III. Although both the FFLO and BCS pairings may occur in regions I, II, and III, the partitions of the preferred pairings in these regions are different. The BCS-like pairing is favored more in region I, while the FFLO-like pairing is favored more in region II. In region III, the BCS and FFLO pairings have equal weighting.

The occurrence of two gaps implies the existence of mixed pairing states in SWSM. The pairing terms in the Hamiltonian, $\quad \sum_{\mathbf{k}, k}\left[\Delta_{F} \psi_{\kappa, \downarrow}(-\mathbf{k}) \psi_{\kappa, \uparrow}(\mathbf{k})+\right.$ $\Delta_{B} \psi_{-\kappa, \downarrow}(-\mathbf{k}) \psi_{\kappa, \uparrow}(\mathbf{k})+$ H.c.], can be rewritten in the form $\sum_{k, \beta}\left[\left(\Delta_{F}+\beta \widetilde{\Delta}_{B}\right) \widetilde{\psi}_{\beta, \Downarrow}(-k) \widetilde{\psi}_{\beta, \Uparrow}(k)+\right.$ H.c. $]$ by rotating the frame reference, where $\widetilde{\psi}_{\beta, \Uparrow}(k)=(1 / \sqrt{2}) e^{i \phi / 2}\left[\left(\cos \frac{\theta}{2} \psi_{+, \uparrow}+\right.\right.$ $\left.\left.\sin \frac{\theta}{2} \psi_{+, \downarrow}\right)+\beta\left(\sin \frac{\theta}{2} \psi_{-, \uparrow}+\cos \frac{\theta}{2} \psi_{-, \downarrow}\right)\right] \quad$ and $\quad \widetilde{\psi}_{\beta, \downarrow}(k)=$ $(1 / \sqrt{2}) e^{-i \phi / 2}\left[\left(\sin \frac{\theta}{2} \psi_{+, \uparrow}-\cos \frac{\theta}{2} \psi_{+, \downarrow}\right)+\beta\left(\cos \frac{\theta}{2} \psi_{-, \uparrow}-\right.\right.$ $\left.\left.\sin \frac{\theta}{2} \psi_{-, \downarrow}\right)\right]$. The spins of the quasiparticle excitations $\widetilde{\psi}_{\beta, \uparrow}^{\dagger}(k)$ and $\widetilde{\psi}_{\beta, \Downarrow}^{\dagger}(k)$ are denoted by $\Uparrow$ and $\Downarrow$. It is found that the quasiparticle excitations are the superposition states of those
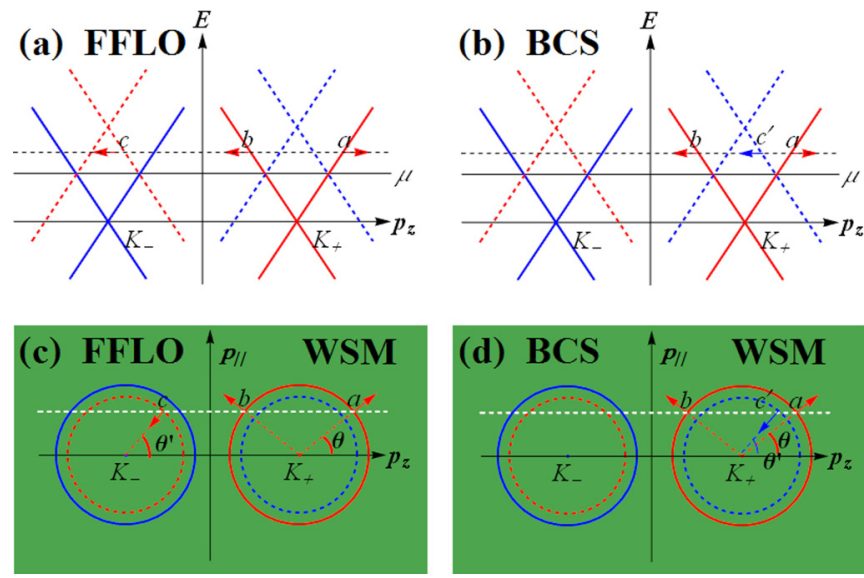

FIG. 2. The energy dispersion of a BdG Hamiltonian. The red (blue) solid lines are the electron part of the Weyl nodes local at $\mathbf{K}_{+}$ $\left(\mathbf{K}_{-}\right)$, and the red (blue) dashed lines are the hole part of the Weyl nodes located at $\mathbf{K}_{+}\left(\mathbf{K}_{-}\right)$. (a) and (b) are for the FFLO and the BCS pairings, respectively, where the directions of the group velocities of the reflected electrons or holes are marked by the arrows. For an electron incident from state $a$, the normal reflected electron is reflected to state $b$, while $\mathrm{AR}$ is reflected to $c$ (for a FFLO pairing) or $c^{\prime}$ (for a BCS pairing). (c) and (d) are the top views of the normal reflected electron and the Andreev reflected hole in the WSM region. The incidence angle and the normal reflection angle are $\theta$, and the AR angle is $\theta^{\prime}$.

states at two Weyl nodes. The parameter $\beta$ plays a significant role in differentiating the quasiparticle excitations $\widetilde{\psi}_{\beta, \sigma}^{\dagger}(k)$.

Figure 1(e) is a snapshot of $\Delta_{F}=0.5 \Delta_{0}$. When the angle is $\theta=\pi / 6, \Delta_{\text {eff }}^{(-)}=0$; that is, one of the gaps is closed. We will see that such closed-gap points will exhibit a distinct feature in AR.

\section{AR IN A HETEROJUNCTION WITH A PERFECT INTERFACE}

In order to reveal its extraordinary features related to the distinctive structure of the superconducting gap, we study AR in an inversion-symmetric doped WSM/SWSM heterojunction with a mixture of BCS and FFLO pairings. Due to the nature of the intranode pairing for FFLO and the internode pairing for BCS, ARs related to the electron-to-hole conversion can occur at different bands. These processes can be understood with Fig. 2. Here the solid red (blue) lines are the bands for the electron part at nodes at $\mathbf{K}_{+}\left(\mathbf{K}_{-}\right)$, and the dashed red (blue) lines are the bands corresponding to the converted hole. More concretely, once an incident electron in the state at node $\mathbf{K}_{\gamma}$ with $k_{z}$ forms a Cooper pair with an electron in the state at node $\mathbf{K}_{\gamma^{\prime}}$ with $\left(\gamma^{\prime}+\gamma\right) K-k_{z}$, a "converted" hole is left in the state at node $\mathbf{K}_{\gamma^{\prime}}$ with $k_{z}-\left(\gamma^{\prime}+\gamma\right) K$. The pairing state has the momentum $\Delta k=\left(\gamma^{\prime}+\gamma\right) K$. Therefore, the Andreev reflected hole is in the state at the same node as that of the incident electron for the FFLO pairing $\left(\gamma^{\prime}=\gamma\right)$, while it is in the state at the node with opposite chirality for the BCS pairing $\left(\gamma^{\prime}=-\gamma\right)$. However, for the mixed pairing in AR, the reflected particles associated with the incident electrons can be in the states of any Weyl node in the WSM. Consequently, 
not only two possible electron reflection processes but also two possible electron-to-hole conversion processes exist at the WSM/SWSM interface. The reflected particle is either within the same Weyl node as that of the incident electron or in a Weyl node different from that of the incident electron. Accordingly, both the BCS- and FFLO-like electron-to-hole conversions can occur at the WSM/SWSM interface.

To understand how the intranode and internode components of ARs work, we first investigate the tunneling process with a perfect interface. We consider an incident electron at node $\mathbf{K}_{+}$ from the WSM side. The directions of the group velocities of the reflected electrons or holes in AR are illustrated in Fig. 2 for the FFLO and BCS pairings. Figures 2(a) and 2(c) are for the FFLO pairing, where the reflected electron and hole appear at the same chirality node (reflected $b$ and $c$ points for the incident $a$ point). Figures 2(b) and 2(d) are for the BCS pairing, where the reflected hole appears in the node with opposite chirality (reflected $c^{\prime}$ point for the incident $a$ point). Because both pairing mechanisms are allowed, the wave functions in the WSM and the SWSM regions can be written in the form

$$
\Psi_{W}=\psi_{+,+}^{W,(e)}+\sum_{\gamma= \pm} r_{\gamma,+}^{(e)} \psi_{\gamma,+}^{W,(e)}+\sum_{\gamma, \kappa= \pm} r_{\gamma, \kappa}^{(h)} \psi_{\gamma, \kappa}^{W,(h)}
$$

for $z<0$ and

$$
\Psi_{S}=\sum_{\gamma= \pm} t_{\gamma,+}^{(e)} \psi_{\gamma,+}^{S,(e)}+\sum_{\gamma= \pm} t_{\gamma,+}^{(h)} \psi_{\gamma,+}^{S,(h)}
$$

for $z>0$. Here $\psi_{\gamma, \kappa}^{W / S,(e / h)}$ are the eigenstates at Weyl nodes $\mathbf{K}_{ \pm}$ in the WSM and the SWSM regions, and $\gamma= \pm$ and $\kappa= \pm$ indicate the chirality and the conduction and valance branches, respectively. The coefficients $r_{\gamma,+}^{(e)}, r_{\gamma,-}^{(h)}, r_{\gamma,+}^{(h)}, t_{\gamma,+}^{(e)}$, and $t_{\gamma,+}^{(h)}$ for the scattered state at Weyl node $\mathbf{K}_{\gamma}$ correspond to the processes taking place in the normal reflection of an electron, AR due to pairing, and transmission of quasiparticles to the SWSM. The specular AR [30] can occur when the pairing potential is larger than the chemical potential, which is not shown in Figs. 2(a) and 2(b). However, the density of states of these states is very small, and the contribution from the specular AR to the conductance is practically negligible.

For an incident electron from Weyl node $\mathbf{K}_{+}$, the normal electron reflection and the AR coefficients are defined as $R_{\gamma,+}^{(e / h)}=\left|r_{\gamma,+}^{(e / h)}\right|^{2}$, where $\gamma= \pm 1$ refer to the reflections falling at $\mathbf{K}_{+}$and $\mathbf{K}_{-}$, respectively. The angle dependence and the energy dependence of the reflection coefficients are shown in Fig. 3. The conservation of the probability-current density is maintained in calculating various transport coefficients. It is found that the intra- and internode components of ARs depend on the incidence angle $\theta$. Such a $\theta$ dependence of $R_{\gamma,+}^{(e / h)}$ manifests the information of the effective gap.

It is found that the $\mathrm{AR}$ coefficients $R_{++}^{h}$ and $R_{-+}^{h}$ are dependent on $E$. If the incident electron has an energy $0<\underset{\widetilde{\Delta}}{E}<$ $\widetilde{\Delta}_{B}-\Delta_{F}\left(\widetilde{\Delta}_{B}>\Delta_{F}\right)$ or $0<E<\Delta_{F}-\widetilde{\Delta}_{B}\left(\Delta_{F}>\widetilde{\Delta}_{B}\right)$, $\mathrm{AR}$ is supported dominantly with the BCS-like pairing or with the FFLO-like pairing, respectively. Figures 3(b) and 3(d) show that the nature of the Andreev reflected states is determined by the relative magnitudes of $\Delta_{F}$ and $\widetilde{\Delta}_{B}$. When we vary this ratio, the reflection can be enhanced at one Weyl node
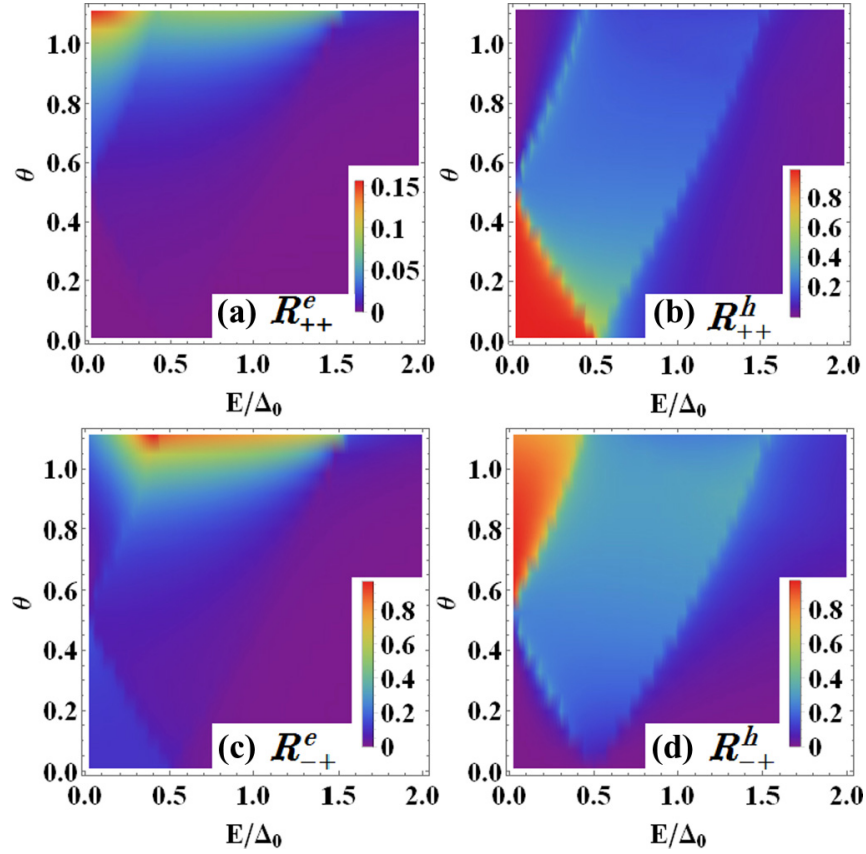

FIG. 3. The transport coefficients as functions of the incident angle $\theta$ and energy $E$ (in units of $\Delta_{0}$ ). (a) $R_{++}^{e}=\left|r_{++}^{e}\right|^{2}$, the normal reflection in $\mathbf{K}_{+}$; (b) $R_{++}^{h}=\left|r_{++}^{h}\right|^{2}$, AR in $\mathbf{K}_{+}$; (c) $R_{-+}^{e}=\left|r_{-+}^{e}\right|^{2}$, the normal reflection in $\mathbf{K}_{-}$; and (d) $R_{-+}^{h}=\left|r_{-+}^{h}\right|^{2}$, AR in $\mathbf{K}_{-}$. The parameters used in the calculations are $\mu=10 \Delta_{0}, \Delta_{F}=0.5 \Delta_{0}$, and $\Delta_{B}=\Delta_{0}$.

and suppressed at another node. For a low energy ( $E$ is very small), AR would occur only at one Weyl node. If the incident electron has an energy $0<E<\Delta_{F}-\widetilde{\Delta}_{B}$ with $\Delta_{F}>\widetilde{\Delta}_{B}$, $A R$ is enhanced at $\mathbf{K}_{-}$, while $A R$ at $\mathbf{K}_{+}$is suppressed. AR with the FFLO-like pairing is supported. However, if the incident electron has an energy $0<E<\widetilde{\Delta}_{B}-\Delta_{F}$ with $\Delta_{F}<\widetilde{\Delta}_{B}$, it dominantly supports AR with the BCS-like pairing. In the limit of the pure BCS or pure FFLO pairing, these results are consistent with previous research. However, if the energy of the incident electron is restricted to $\Delta_{\text {eff }}^{(-)}<E<\Delta_{\text {eff }}^{(+)}$, the mixed pairing in AR takes effect, and ARs are apportioned between the BCS and FFLO states.

The total reflection is given by the sums of intra- and internode ARs. Comparing Figs. 3(b) and 3(d) with Fig. 1(e), the four regions of AR can be directly correlated to the four regions of effective gap. The existence of two gaps can result in different manifestations of tunneling in a WSM/SWSM hybrid with AR. Because $\Delta_{\text {eff }}^{(+)}>\Delta_{\text {eff }}^{(-)}$, both gaps take effect if the energy of incident electrons is smaller than $\Delta_{\text {eff }}^{(-)}$. However, only $\Delta_{\text {eff }}^{(+)}$takes effect if $\Delta_{\text {eff }}^{(-)}<E<\Delta_{\text {eff }}^{(+)}$. Therefore, if $\Delta_{\text {eff }}^{(-)}<E<\Delta_{\text {eff }}^{(+)}$, only half of the incident electrons are paired and undergo $\mathrm{AR}$, while the other half are transmitted into the superconducting region directly. The total reflection equals $1 / 2$. We refer to such a phenomenon as the nonholonomic AR. We also see from Fig. 3 that $R_{++}^{h}$ and $R_{-+}^{h}$ are small for the energy $E>\left|\Delta_{F}+\tilde{\Delta}_{B}\right|$. The electron energy is now outside the energy gap, and the effective pairing is suppressed. As the gap is closed, $\Delta_{F}=\widetilde{\Delta}_{B}$, ARs alternate at $E=0$ and $\theta \simeq \pi / 6$. 


\section{THE INFLUENCE OF INTERFACE POTENTIALS ON AR}

\section{A. Two possible interface scattering potentials}

Two possible scattering processes exist at the WSM/SWSM interface: (i) the reflected states are in the same Weyl node as that of incident electrons, and (ii) the reflected states are in a Weyl node different from that of incident electrons. Here we consider two kinds of barrier potentials at the interface, the intra-SIP and inter-SIP.

In general, the intra-SIP can be written in the form

$$
V_{I}=U_{I}\left(\begin{array}{cc}
\tau_{0} \sigma_{0} & 0 \\
0 & -\tau_{0} \sigma_{0}
\end{array}\right) \delta(z)
$$

with a strength of $U_{I}$. The matrix $\tau_{0}$ describing the space of the Weyl node implies that the intra-SIP conserves the chirality in the scattering. The inter-SIP takes the form

$$
V_{R}=U_{R}\left(\begin{array}{cc}
\tau_{x} \sigma_{0} & 0 \\
0 & -\tau_{x} \sigma_{0}
\end{array}\right) \delta(z),
$$

with a strength of $U_{R}$. The matrix $\tau_{x}$ implies the chirality is reversed in the scattering.

\section{B. The boundary conditions of the intra-SIP and the inter-SIP}

As pointed out in Ref. [28], the boundary condition with a finite interface potential should be specifically formulated if the particle has a linear energy dispersion. We multiply both sides of the BdG equation by $\left(i / \hbar v_{F}\right) I_{2 \times 2} \otimes \tau_{z} \sigma_{z}$ and obtain $\partial_{z} \Psi+i \widehat{D} \Psi=-i \widehat{A} \delta(z) \Psi$, where

$$
\widehat{D}=\left(\begin{array}{cc}
d_{x y}^{(+)} & 0 \\
0 & d_{x y}^{(-)}
\end{array}\right)
$$

and

$$
\widehat{A}=\left(\begin{array}{cc}
\tau_{z} \sigma_{z} Z+i \tau_{y} \sigma_{z} Z^{\prime} & 0 \\
0 & \tau_{z} \sigma_{z} Z+i \tau_{y} \sigma_{z} Z^{\prime}
\end{array}\right) .
$$

Here $Z=U_{I} / \hbar v_{F}$ and $Z^{\prime}=U_{R} / \hbar v_{F}, d_{x y}^{( \pm)}$are operators given by $d_{x y}^{( \pm)}=(i / \hbar) \tau_{z}\left(\sigma_{y} p_{x}-\sigma_{x} p_{y}\right)+\left(\mu / \hbar v_{F}\right) \tau_{z} \sigma_{z} \pm$ $\left(1 / \hbar v_{F}\right) \tau_{z}\left[b \sigma_{0}-E \sigma_{z}+\Delta^{T}\right], \Delta^{T}=\theta(z)\left(\Delta \sigma_{+}+\Delta^{*} \sigma_{-}\right)$, and $\sigma_{ \pm}=\left(\sigma_{x} \pm i \sigma_{y}\right) / 2$. Integrating over a small region $[-\delta, \delta]$ crossing the interface at $z=0$, it is found that

$$
\Psi(\delta)=\hat{P}_{z} \exp \left[-i \int_{-\delta}^{\delta} d z(\widehat{A} \delta(z)+\widehat{D})\right] \Psi(-\delta),
$$

where $\hat{P}_{z}$ is the spatial ordering operator. In the limit of $\delta \rightarrow 0$, we obtain the boundary condition

$$
\Psi\left(0_{+}\right)=\exp (-i \widehat{A}) \Psi\left(0_{-}\right) .
$$

Now we use this relation to obtain the boundary condition for the intra-SIP. It can be written in the form

$$
\Psi\left(0_{+}\right)=\exp \left[-i Z \Sigma_{z} \otimes\left(\tau_{z} \sigma_{z}\right)\right] \Psi\left(0_{-}\right),
$$

where

$$
\Sigma_{z}=\left(\begin{array}{cc}
1 & 0 \\
0 & -1
\end{array}\right)
$$

The operator $\exp \left[-i Z \Sigma_{z} \otimes\left(\tau_{z} \sigma_{z}\right)\right]$ can be diagonalized with the diagonal elements $e^{ \pm i Z}$. Therefore, the effect of the intraSIP on the wave function crossing the interface is to modify the phase. This implies that the intra-SIP is transparent for massless Weyl fermions at some values of the intra-SIP strength. The reason behind such a phenomenon arises ultimately from the fact that the Weyl fermion has a definite chirality and the intra-SIP is chirality independent.

The boundary condition for the inter-SIP is given as

$$
\Psi\left(0_{+}\right)=I_{2 \times 2} \otimes \Lambda \Psi\left(0_{-}\right),
$$

where $\Lambda=\tau_{0} \sigma_{0} \cosh Z^{\prime}+\tau_{y} \sigma_{z} \sinh Z^{\prime}$. The operator $\Lambda$ indicates explicitly the node exchange. It results in a masslike term in the Dirac equation [31]. Correspondingly, the chirality of the Weyl fermion is no longer conserved.

\section{AR in WSM/SWSM heterojunctions with interface potentials}

Now we investigate the carrier transport in a WSM/SWSM heterojunction with the intra-SIP and the inter-SIP. The following parameters are used in our calculations: $\Delta_{F}=\Delta_{0}$, $\Delta_{B}=0.5 \Delta_{0}$, and $\theta=\pi / 6$.

\section{Intra-SIP}

Since the effect of the intra-SIP is only to modify the phase of wave functions, the transport coefficients are periodic functions of $Z$ with a period $\pi$. Figures 4(a)-4(d) depict $R_{\gamma,+}^{(e)}$ and $R_{\gamma,+}^{(h)}$ for an electron incident at the Weyl point $\mathbf{K}_{+}$. Two remarkable features are observed. First, as expected, AR is suppressed with increasing intra-SIP strength. At the same time, the normal electron reflection is enhanced. Second, the intra-SIP strength $Z$ can affect the intra- and internode components in ARs. Figures 4(a)-4(d) show that when the energies are at the edges of the effective gap, $\left|\Delta_{F}-\widetilde{\Delta}_{B}\right|$ and $\left|\Delta_{F}+\widetilde{\Delta}_{B}\right|, R_{-,+}^{(e)}, R_{+,+}^{(h)}$, and $R_{-,+}^{(h)}$ have peaks, but $R_{+,+}^{(e)}$ is at the minimum. Away from the gap edges, the electron is reflected in favor of its former state. The magnitudes of the intra- and internode ARs are affected by the intra-SIP strength $Z$, as shown in Figs. 4(a)-4(d). ARs stemming from both superconducting pairings would be suppressed with increasing $Z$. Therefore, those stemming from the FFLO pairing are suppressed much more than that of the BCS pairing. The reason is that the FFLO pairing needs a momentum shift in the formation of a Cooper pair. However, the intra-SIP barrier reduces the possibility of such a shift in tunneling.

\section{Inter-SIP}

The energy-dependent $R_{\gamma,+}^{(e)}$ and $R_{\gamma,+}^{(h)}$ for the inter-SIP are shown in Figs. 4(e)-4(h). Because the scattering at the interface is accompanied by a chirality change, the BCS-pairingmediated ARs increase, and the FFLO-pairing-mediated ARs decrease when the inter-SIP strength increases. For an incident energy with values at the edges of the effective gaps, the electron reflections are reduced, and ARs are increased.

\section{CONDUCTANCE IN THE WSM/SWSM HETEROJUNCTION}

The conductance can be calculated by the formula

$$
\frac{G^{\mathrm{tot}}}{G_{0}}=\frac{\int_{0}^{\pi / 2} \sin \theta d \theta G(\theta)}{\int_{0}^{\pi / 2} \sin \theta d \theta G_{0}(\theta)}
$$



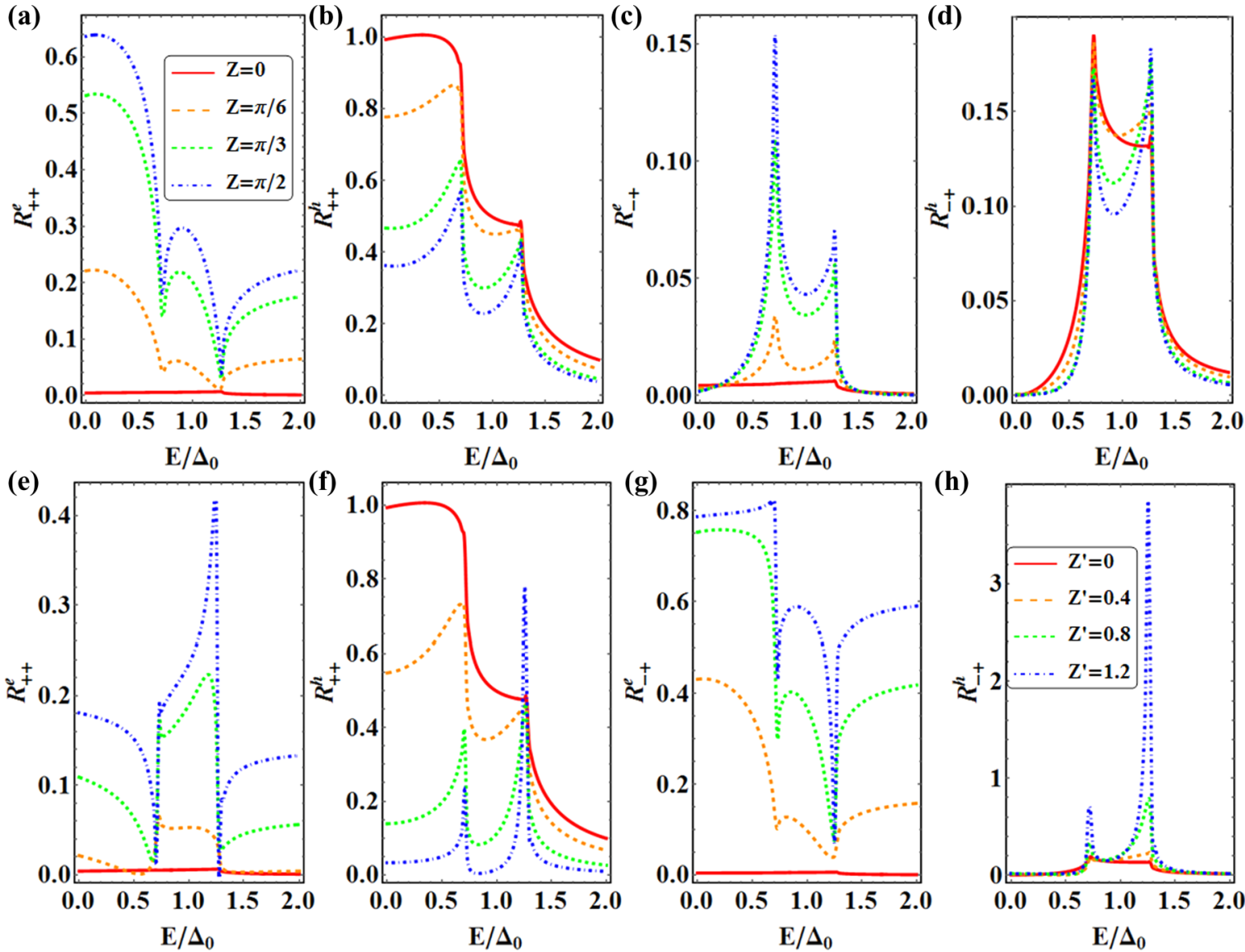

FIG. 4. The influence of the interface configurations on the transport coefficients (a)-(d) for the intra-SIP and (e)-(h) for the inter-SIP. (a) and (e) The normal reflection in $\mathbf{K}_{+}$, (b) and (f) AR in $\mathbf{K}_{+}$, (c) and (g) the normal reflection in $\mathbf{K}_{-}$, and (d) and (h) AR in $\mathbf{K}_{-}$. The parameters used in the calculations are $\theta=\pi / 6, \mu=10 \Delta_{0}, \Delta_{F}=\Delta_{0}, \Delta_{B}=0.5 \Delta_{0}$.

where $G_{0}$ is the conductance for a normal WSM/WSM heterojunction with an appropriate interface potential, $G(\theta)=$ $\int_{0}^{2 \pi} d \phi\left[\cos \theta\left(1-\sum_{\gamma \gamma^{\prime}} R_{\gamma^{\prime}, \gamma}^{(e)}\right)+\sum_{\gamma \gamma^{\prime}} \cos \theta^{\prime} R_{\gamma^{\prime}, \gamma}^{(h)}\right]$ and $G_{0}(\theta)=\int_{0}^{2 \pi} d \phi \cos \theta\left(\left|t_{1}\right|^{2}+\left|t_{2}\right|^{2}\right)$ are the angle-resolved conductances, $t_{1}$ and $t_{2}$ are the normal transmission coefficients for a normal WSM/WSM heterojunction, and $\theta^{\prime}$ is the AR angle [as shown in Figs. 2(a) and 2(b)], which is little different from the incident angle of electron $\theta$. In a practical application, we always have $\mu \gg \Delta_{0}$. The Andreev approximation can be used so that we take $\theta^{\prime}=\theta$ in our calculations.

The energy dependence of $G(\theta) / G_{0}(\theta)$ for a perfect interface is shown in Fig. 5. The ratio $G(\theta) / G_{0}(\theta)$ exhibits a threestep structure in regard to the energy and the incident angle. The sharp drop occurs at $E=|0.5 \pm \sin \theta| \Delta_{0}$. When the energy of incident electrons is in the range $E<\left|\Delta_{F}-\widetilde{\Delta}_{B}\right|$, the energy is within a full gap. The electron-to-hole conversion results in a hole being reflected. As a consequence, $G(\theta) \simeq 2 G_{0}(\theta)$. When the energy exceeds the effective gap $\left|\Delta_{F}-\widetilde{\Delta}_{B}\right|$, the paired and unpaired states each account for half of the incident electrons. Part of the electrons could not feel the gap $\left|\Delta_{F}-\widetilde{\Delta}_{B}\right|$, so they are not actively involved in AR. As a result of the nonholonomic AR, $G(\theta)$ drops to $(3 / 2) G_{0}(\theta)$. When the energy is far away from the Fermi energy, both effective gaps are suppressed, and no AR takes place. The ratio $G(\theta) / G_{0}(\theta)$ approximately equals 1 . From the above analysis, the three-step structure in the angle-resolved conductance is apparent as a consequence of the excitation spectrum properties for the SWSM with mixed BCS and FFLO superconducting pairings.

The influence of the intra-SIP and the inter-SIP on the total conductivities is shown in Fig. 6. Figures 6(a) and 6(d) clearly

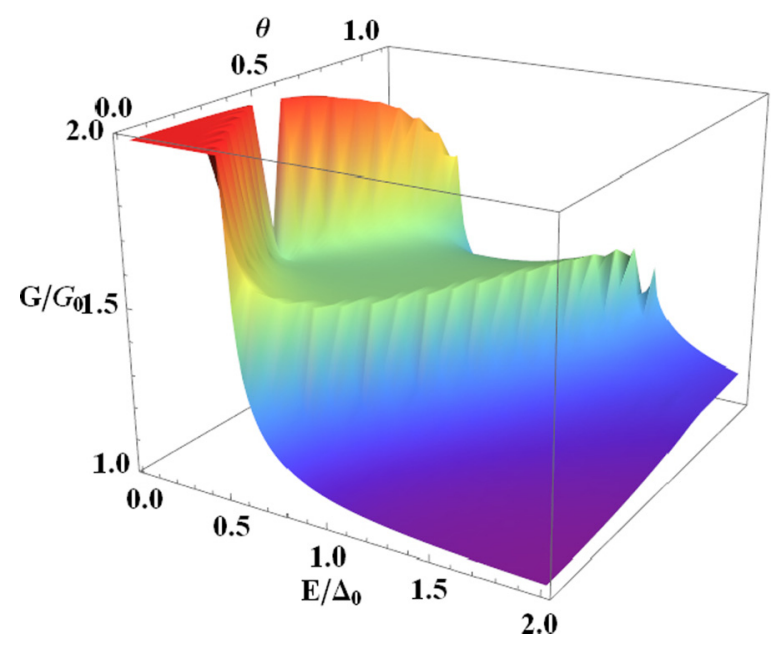

FIG. 5. The angle-resolved conductance. The parameters used in the calculations are $\mu=10 \Delta_{0}, \Delta_{F}=0.5 \Delta_{0}$, and $\Delta_{B}=\Delta_{0}$. 

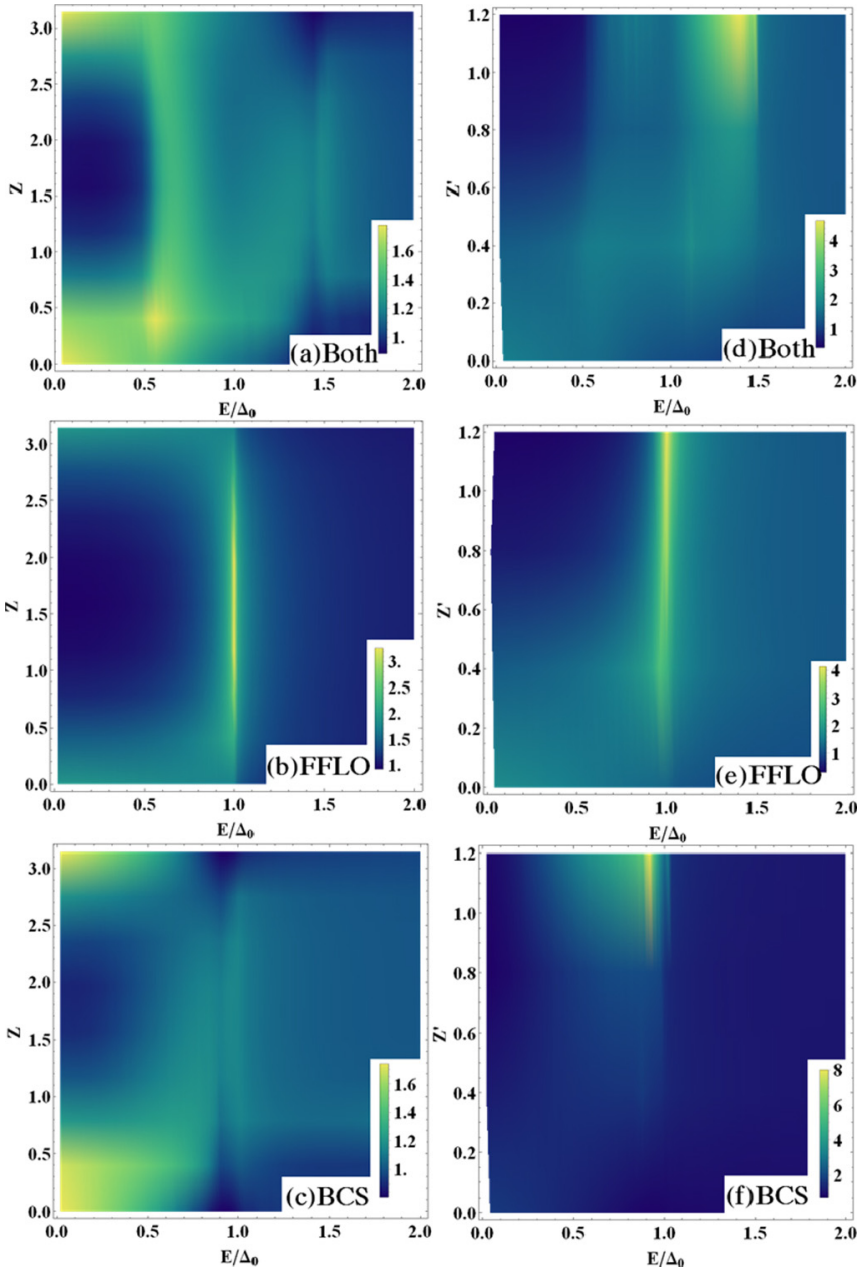

FIG. 6. Contour plot of the ratio $G^{\text {tot }} / G_{0}$ with different interface configurations (a)-(c) for the intra-SIP and (d)-(f) for the inter-SIP. (a) and (d) The co-occurrence of the BCS and FFLO superconducting pairings $\left(\Delta_{F}=\Delta_{0}\right.$ and $\left.\Delta_{B}=0.5 \Delta_{0}\right)$, (b) and (e) the pure FFLO superconducting pairings $\left(\Delta_{F}=\Delta_{0}\right.$ and $\left.\Delta_{B}=0\right)$ and (c) and (f) the pure BCS superconducting pairings $\left(\Delta_{F}=0\right.$ and $\left.\Delta_{B}=\Delta_{0}\right)$. The chemical potential is taken as $\mu=10 \Delta_{0}$.

display evidence of the effective gap structure. In Fig. 6(a), the conductance varies periodically with the change in the intraSIP strength for $E \leqslant\left|\Delta_{F}-\Delta_{B}\right|$ and becomes slowly varying for $\left|\Delta_{F}-\Delta_{B}\right|<E \leqslant\left|\Delta_{F}+\Delta_{B}\right|$. However, Fig. 6(d) shows an exponentially dwindling conductance for the inter-SIP boundary condition when $E \leqslant\left|\Delta_{F}-\Delta_{B}\right|$. The ratio $G^{\text {tot }} / G_{0}$ is enhanced with increasing inter-SIP strength. $G_{0}$ is exponentially depressed when $\left|\Delta_{F}-\Delta_{B}\right|<E \leqslant\left|\Delta_{F}+\Delta_{B}\right|$. In this region, the inter-SIP increases the internode AR. For $E>\left|\Delta_{F}+\Delta_{B}\right|$, the energy of the incident electron is outside the gap, and the pairings are not the primary avenue for tunneling. The tunneling progresses like that in a WSM/WSM heterojunction, and the conductance reaches its value of $G_{0}$. For comparison, we also plot the total conductances with only the FFLO pairing in Figs. 6(b) and 6(e) and with only the BCS pairing in Figs. 6(c) and 6(f). These plots show a periodic dependence on $Z$ in the conductances for the intra-SIP and exponential dwindling with $Z^{\prime}$ for the inter-SIP. Figures 6(b) and 6(e) unambiguously display the gap edge $\Delta_{F}$ for the FFLO pairing. However, because the effective gap $\Delta_{B}$ for the BCS pairing is dependent on the incident angle, the gap edge in Figs. 6(c) and 6(f) is not obvious for a weak inter-SIP.

\section{SUMMARY}

In summary, the superconducting states mediated by both the BCS and the FFLO pairings are studied in the SWSM. The coexistence of two pairing mechanisms not only shifts the chemical potential but also gives rise to an anisotropic effective pairing potential. We showed that the spectra of the SWSM exhibits a distinctive two-gap structure. In the investigation of ARs in a WSM/SWSM heterojunction, we found that the intra- and internode ARs increase significantly when the energy of the incident electron takes a value between the two edges of the effective gap, particularly for the nonholonomic AR. The incident-angle dependence of the transport coefficients displays the structure of the effective superconducting gap. We showed that the mixture of BCS and FFLO pairings leads to a three-step structure of the conductance with respect to the energy and the incident angle, which starts at 2 when the energy is below the lower edge of the effective gap $\Delta_{\text {eff }}^{(-)}$, drops to $3 / 2$ for $\Delta_{\text {eff }}^{(-)}<E<\Delta_{\text {eff }}^{(+)}$due to a nonholonomic AR, and then drops to 1 when the energy is larger than $\Delta_{\text {eff }}^{(+)}$. To investigate the effect of the interface scattering on transport coefficients, we proposed two different interface potentials (the intra-SIP and the inter-SIP) and analyzed their influence on ARs. We showed that the conductance is a periodic function of the strength of the intra-SIP and an exponential function of the strength of the inter-SIP. The results indicated that the conductance can be used to determine which mechanism dominates the pairing in the SWSM. Recently, the unconventional superconductivity was discovered experimentally by using a hard point contact on Weyl semimetal TaAs crystals [32,33]. The experimental setup with the point contact between the tip and the Weyl semimetal can be thought of as a kind of WSM/SWSM heterojunction. Based on a similar setup, one could investigate the novel superconducting states based on Weyl materials and demonstrate the property of SWSMs, such as a superconducting gap in the bulk state. Modulation of the point contact may provide an effective method for understanding the scattering features.

\section{ACKNOWLEDGMENTS}

The authors are thankful for the support from NSFC (Grants No. 11274013 and No. 11774006), NBRP of China (2012CB921300), and an Australian Research Council Discovery Grant (Grant No. DP160101474). We thank D. Fisher for a critical reading of the manuscript.
[1] G. Y. Cho, J. H. Bardarson, Y.-M. Lu, and J. E. Moore, Phys. Rev. B 86, 214514 (2012).
[2] T. Meng and L. Balents, Phys. Rev. B 86, 054504 (2012).

[3] H. Wei, S.-P. Chao, and V. Aji, Phys. Rev. B 89, 014506 (2014). 
[4] Y. Sun, S.-C. Wu, M. N. Ali, C. Felser, and B. Yan, Phys. Rev. B 92, 161107 (2015).

[5] Y. Qi, P. G. Naumov, M. N. Ali, C. R. Rajamathi, O. Barkalov, M. Hanfland, S.-C. Wu, C. Shekhar, Y. Sun, V. Süb, M. Schmidt, E. Pippel, P. Werner, R. Hillebrand, T. Förster, E. Kampertt, W. Schnelle, S. Parkin, R. J. Cava, C. Felser, B. Yan, and S. A. Medvedev, Nat. Commun. 7, 11038 (2016).

[6] R. Joynt and L. Taillefer, Rev. Mod. Phys. 74, 235 (2002).

[7] Y. Yanase, Phys. Rev. B 94, 174502 (2016).

[8] G. Bednik, A. A. Zyuzin, and A. A. Burkov, Phys. Rev. B 92, 035153 (2015).

[9] T. Zhou, Y. Gao, and Z. D. Wang, Phys. Rev. B 93, 094517 (2016).

[10] P. Hosur, X. Dai, Z. Fang, and X.-L. Qi, Phys. Rev. B 90, 045130 (2014).

[11] R. Wang, L. Hao, B. Wang, and C. S. Ting, Phys. Rev. B 93, 184511 (2016).

[12] M. D. Bachmann, N. Nair, F. Flicker, R. Ilan, T. Meng, N. J. Ghimire, E. D. Bauer, F. Ronning, J. G. Analytis, and P. J. W. Moll, Sci. Adv. 3, e1602983 (2017).

[13] L. Hao, R. Wang, P. Hosur, and C. S. Ting, Phys. Rev. B 96, 094530 (2017).

[14] J. Bardeen, L. N. Cooper, and J. R. Schrieffer, Phys. Rev. 108, 1175 (1957).

[15] P. Fulde and R. A. Ferrell, Phys. Rev. 135, A550 (1964).

[16] A. I. Larkin and Y. N. Ovchinnikov, Zh. Eksp. Teor. Fiz. 47, 1136 (1964) [Sov. Phys. JETP 20, 762 (1965)].

[17] J. P. A. Devreese and J. Tempere, Phys. Rev. A 89, 013616 (2014).

[18] A. Andreev, Zh. Eksp. Teor. Fiz. 46, 1823 (1964) [Sov. Phys. JETP 46, 1823 (1964)].
[19] G. E. Blonder, M. Tinkham, and T. M. Klapwijk, Phys. Rev. B 25, 4515 (1982).

[20] Q. Cui, C.-R. Hu, J. Y. T. Wei, and K. Yang, Phys. Rev. B 85, 014503 (2012).

[21] W. Chen, L. Jiang, R. Shen, L. Sheng, B. Wang, and D. Xing, Europhys. Lett. 103, 27006 (2013).

[22] S. Uchida, T. Habe, and Y. Asano, J. Phys. Soc. Jpn. 83, 064711 (2014).

[23] H. Burkhardt and D. Rainer, Ann. Phys. (Berlin, Ger.) 506, 181 (1994).

[24] K. Yang and D. F. Agterberg, Phys. Rev. Lett. 84, 4970 (2000).

[25] Y. Kim, M. J. Park, and M. J. Gilbert, Phys. Rev. B 93, 214511 (2016).

[26] N. Bovenzi, M. Breitkreiz, P. Baireuther, T. E. O’Brien, J. Tworzydło, İ. Adagideli, and C. W. J. Beenakker, Phys. Rev. B 96, 035437 (2017).

[27] D.-L. Deng, S.-T. Wang, and L.-M. Duan, Phys. Rev. B 89, 075126 (2014).

[28] S. Ryu, A. P. Schnyder, A. Furusaki, and A. W. W. Ludwig, New J. Phys. 12, 065010 (2010).

[29] T. Meng and L. Balents, Phys. Rev. B 96, 019901(E) (2017).

[30] C. W. J. Beenakker, Phys. Rev. Lett. 97, 067007 (2006).

[31] G. J. Clerk and B. H. J. McKellar, Phys. Rev. C 41, 1198 (1990).

[32] H. Wang, H. C. Wang, H. W. Liu, H. Lu, W. H. Yang, S. Jia, X. J. Liu, X. C. Xie, J. Wei, and J. Wang, Nat. Mater. 15, 38 (2016).

[33] H. Wang, H. C. Wang, Y. Q. Chen, J. W. Luo, Z. J. Yuan, J. Liu, Y. Wang, S. Jia, X. J. Liu, J. Wei, and J. Wang, Sci. Bull. 62, 425 (2017). 